\title{
Arginine as an adjuvant to chemotherapy improves clinical outcome in active tuberculosis
}

\author{
T. Schön*, D. Elias ${ }^{\#, \uparrow, ~ F . ~ M o g e s ~}{ }^{+}$, E. Melese ${ }^{+}$, T. Tessema ${ }^{+}$, O. Stendahl*, S. Britton ${ }^{\S}$, T. Sundqvist*
}

Arginine as an adjuvant to chemotherapy improves clinical outcome in active tuberculosis. T. Schön, D. Elias, F. Moges, E. Melese, T. Tessema, O. Stendahl, S. Britton, T. Sundqvist. (C) ERS Journals Ltd 2003.

ABSTRACT: Nitric oxide (NO) is involved in the host defence against tuberculosis (TB). Patients with TB exhibit increased catabolism and reduced energy intake. Thus the hypothesis for this study was that restoring a relative deficiency in the amino acid arginine, the substrate for mycobactericidal NO production, would improve the clinical outcome of TB by increasing NO production.

In a randomised double-blind study, patients with smear-positive $T B(n=120)$ were given arginine or placebo for 4 weeks in addition to conventional chemotherapy. Primary outcomes were sputum conversion, weight gain, and clinical symptoms after week 8. Secondary outcomes were sedimentation rate and levels of NO metabolites, arginine, citrulline, and tumour necrosis factor- $\alpha$.

Compared with the human immunodeficiency virus (HIV)-ITB+ placebo group, the $\mathrm{HIV}-/ \mathrm{TB}+$ patients in the arginine group showed significant improvement, defined as increased weight gain, higher sputum conversion rate and faster reduction of symptoms, such as cough. The arginine level increased after week 2 in the HIV-/TB + arginine group (100.2 $\mu \mathrm{M}$ (range 90.5-109.9) versus 142.1 $\mu \mathrm{M}$ (range 114.1-170.1)) compared with the HIV-ITB+ placebo group $(105.5 \mu \mathrm{M}$ (range 93.7-117.3) versus $95.7 \mu \mathrm{M}$ (range 82.4-108.9)). HIV seroprevalence was $52.5 \%$. No clinical improvement or increase in serum arginine was detected in arginine supplemented $\mathrm{HIV}+I \mathrm{~TB}+$ patients compared with placebo.

Arginine is beneficial as an adjuvant treatment in human immunodeficiency virusnegative patients with active tuberculosis, most likely mediated by increased production of nitric oxide.

Eur Respir J 2003; 21: 483-488.
*Dept of Medical Microbiology, Faculty of Health Sciences, Linköping University, Linköping, ${ }^{\S}$ Dept of Infectious Diseases, Karolinska Hospital, Stockholm, and Microbiology and Tumour Biology Centre, Karolinska Institute, Stockholm, Sweden. ${ }^{\#}$ Armauer Hansen Research Institute (AHRI), Addis Ababa, and ${ }^{+}$Gondar College of Medical Sciences (GCMS), Gondar, Ethiopia.

Correspondence: T. Schön, Dept of Medical Microbiology, Faculty of Health Sciences, Linköping University, SE-581 85 Linköping, Sweden.

Fax: 4613224789

E-mail: t.schon@telia.com

Keywords: Arginine, human immunodeficiency virus, Mycobacterium tuberculosis, nitric oxide, tuberculosis, tumour necrosis factor- $\alpha$

Received: October 22002

Accepted after revision: November 14 2002

This work was supported by SAREC (Swe-1999-267), the Swedish Heart and Lung Foundation (20041594) and the Swedish Research Council.
Tuberculosis (TB), which is caused by Mycobacterium tuberculosis, is an important global health problem that is aggravated by associated factors, such as co-infection with human immunodeficiency virus (HIV) and increasing multidrug resistance [1]. Of the estimated 1.7 billion people infected with $M$. tuberculosis, only $5-10 \%$ progress to clinically active disease, indicating effective host defence mechanisms [1]. $\mathrm{HIV}+$ individuals are at increased risk of TB, which strongly suggests involvement of CD4+ T-helper type-1 (Th1) cells in protective immunity against $M$. tuberculosis. In murine models of TB, an initial Th1 response with high levels of cytokines, such as tumour necrosis factor (TNF)- $\alpha$ and interferon (IFN)$\gamma$, is followed by a T-helper type- 2 response, which limits the inflammatory response [2].

Numerous reports based on animal models suggest that nitric oxide (NO) is important for host resistance during the acute phase of TB [3-5]. The role of NO in humans is controversial, although recent findings indicate involvement in human TB [5-10]. Inducible NO synthase (iNOS) catalyses the synthesis of NO and citrulline from L-arginine in macrophages activated by cytokines, such as TNF- $\alpha$ and IFN- $\gamma$ $[6,11]$. NO is highly unstable and decays to its stable end products nitrate and nitrite, which are eliminated in the urine [11]. L-arginine is a semi-essential amino acid that can become essential in some diseases and under certain circumstances [12]. Normal plasma levels of L-arginine are maintained mainly through dietary intake and synthesis from citrulline in the kidney [12].

In as much as malnutrition and reduced food intake are associated with TB [13], the hypothesis for this study was that patients with this disease would be prone to developing arginine deficiency, which could limit one of the major mycobactericidal pathways, iNOS catalysed production of $\mathrm{NO}$ from arginine. The patients were subdivided according to HIV serology, because HIV affects cell-mediated immunity, which is clearly linked to iNOS-mediated NO production [6, $11,14,15]$. A placebo-controlled randomised trial at Gondar Hospital in Gondar, Ethiopia, was conducted to ascertain whether adjuvant arginine supplementation can improve the clinical outcome of pulmonary TB.

\section{Methods}

\section{Patients}

Patients with newly diagnosed smear-positive TB, presenting consecutively, were recruited with informed consent 
from December 2000-December 2001 at the Direct Observed Treatment Short-Course (DOTS) Clinic at Gondar Hospital. The inclusion criteria were an age of 15-60 yrs and acid fast bacilli (AFB) smear-positive TB by microscopy, using ZiehlNieelsen staining, as recommended by the World Health Organization (WHO) for DOTS [1]. The exclusion criteria were hospitalisation, pregnancy or clinical signs of any concomitant disease, such as diabetes mellitus, acute renal failure or infectious diseases other than TB/HIV. Smear positivity was defined as two of three positive morning sputum samples or one of three positive with a chest radiograph and clinical symptoms suggestive of pulmonary TB. HIV status was evaluated by serology (Enzygnost, Behring, Germany) and an agglutination test (HIV spot; Genelabs diagnostics, Singapore).

Sedimentation rate (SR; Sedistainer, Beckton Dickinson, San Jose, CA, USA) and sputum AFB status by microscopy were recorded at baseline and 2 and 8 weeks after initiating treatment. Sputum conversion was defined as three consecutive sputum smears negative for AFB. Weight was recorded at baseline and 1, 2, 4 and 8 weeks after beginning treatment. Blood and urine samples for laboratory analyses were obtained at baseline and 2 and 8 weeks after starting treatment. Serum $\mathrm{TNF}-\alpha$ levels were analysed using Quantikine high-sensitivity assay according to the manufacturer's descriptions (R\&D Diagnostics, Minneapolis, MN, USA).

Using a standard form, patients were initially interviewed regarding duration of clinical symptoms (haemoptysis, cough, chest pain, fever, and night sweating), and at weeks 2 and 8 concerning the presence or absence of symptoms. The study was approved by the ethics committees at the Gondar College of Medicine, Gondar, Ethiopia, the Faculty of Health Sciences, Linköping, Sweden, and the Ethiopian Science and Technology Commission, Addis Ababa, Ethiopia.

\section{Treatment regimes}

All treatment was done on an outpatient basis, according to the Ethiopian National Guidelines for DOTS treatment of smear-positive pulmonary TB (based on WHO recommendations). The chemotherapy consisted of isoniazid, pyrazinamide, rifampicin and streptomycin or ethambutol during the intensive phase of 2 months followed by isoniazid and ethambutol for 6 months. All drugs, including arginine or placebo were administered orally supervised daily except for streptomycin which was given subcutaneously. At initiation of anti-TB therapy, patients with TB were assigned by randomisation in blocks of six (performed by the State Pharmacy of Sweden, Stockholm, Sweden), to supplementation with identical capsules of $1 \mathrm{~g}$ argine or $1 \mathrm{~g}$ placebo (State Pharmacy of Sweden) daily, administered orally for 4 weeks. The primary outcomes were sputum conversion, weight gain, and clinical symptoms after week 8 . The study was double blinded and a sealed copy of the treatment code was kept by the project leader until all data had been collected and analysed.

\section{Analysis of urinary levels of nitrite and nitrate}

The sum of nitrate and nitrite concentration in urine was determined essentially as described by VERDON et al. [16]. A urine sample was diluted in distilled water and incubated with nitrate reductase from Aspergillus $\left(1 \mathrm{U} \cdot \mathrm{mL}^{-1}\right)$, nicotinamide adenine dinucleotide phosphate ( $1 \mu \mathrm{M}$; Sigma, St Louis, MO, USA), glucose-6-phosphate (0.5 mM, Sigma), and glucose-6dehydrogenase $\left(0.16 \mathrm{U} \cdot \mathrm{mL}^{-1}\right.$, Sigma) in phosphate-buffered saline for $45 \mathrm{~min}$ at room temperature. Thereafter, the nitrite level was determined by the Griess reaction, adding first sulphanilic acid $\left(2 \mathrm{mg} \cdot \mathrm{mL}^{-1}\right)$ in $\mathrm{H}_{2} \mathrm{PO}_{4}(15 \mathrm{mM})$ and then $N$ (1-naphtyl) ethylenediamine $\left(1 \mathrm{mg} \cdot \mathrm{mL}^{-1}\right)$. The urine samples were then analysed in an enzyme-linked immunosorbent assay reader at $540 \mathrm{~nm}$. The recorded values were transposed to a standard curve.

\section{Analysis of serum L-arginine and citrulline by high- performance liquid chromatography}

All serum samples were filtered by centrifuging at $13,000 \times g$ for $90 \mathrm{~min}$ in a Microcon-3 tube (Amicon Inc., Beverly, USA) with a cut-off of 3,000 D and subsequently stored at $-20^{\circ} \mathrm{C}$ until analysed. Serum L-arginine and citrulline were analysed using a modified version of the protocol described by CARLBERG [17]. The high-performance liquid chromatography (HPLC) system consisted of an Optilab 931 pump (Shimadzu, Tokyo, Japan) and an RF-535 Fluorescence HPLC monitor (Shimadzu), equipped with a $5 \mu \mathrm{m}$ Microsphere C18 column $(250 \times 4 \mathrm{~mm})$ from Knauer (Berlin, Germany). An excitation/ emission wavelength of $338 / 425 \mathrm{~nm}$ was used. A mobile phase comprising $7.5 \%$ acetonitrile, $7.5 \%$ methanol (Fisher Scientific, Leichestershire, UK), and $0.42 \%$ tetrahydrofuran (Sigma) in $10 \mathrm{mM} \mathrm{KH} \mathrm{KO}_{4}$ was used at a flow rate of $1 \mathrm{~mL} \cdot \mathrm{min}^{-1}$. The HPLC column was washed with acetonitrile:methanol: tetrahydrofuran in a ratio of 30:30:2.5 in $10 \mathrm{mM} \mathrm{KH}_{2} \mathrm{PO}_{4}$. Precolumn derivatisation of samples was performed with an equal volume of o-pthaldialdehyde reagent solution (Sigma). Levels of serum L-arginine and citrulline were transposed from a standard curve constructed from known concentrations of L-arginine and citrulline (Sigma).

\section{Statistics}

Normality of distribution was checked by inspecting frequency plots $\left(Q^{\prime} / Q^{\prime}\right.$ plots $)$. Effects of arginine treatment, compared with placebo, on primary and secondary outcomes in HIV- and HIV+ individuals, respectively, were evaluated by double multivariate repeated measures analysis. Differences between the arginine- and placebo-supplemented groups from weeks $0-8$ were also evaluated separately for each variable using repeated measures analysis of variance for continuous variables and Kruskal-Wallis analysis for discrete variables. Continuous data are expressed as means with $95 \%$ confidence intervals (CI). A p-value of $<0.05$ was regarded as statistically significant.

\section{Results}

\section{Patients}

Of 184 eligible patients, 120 sputum AFB-positive patients were included in the study and 64 were excluded due to hospitalisation or because they lived too far away from the study area to attend the DOTS programme. Total seroprevalence of HIV in the study population was $52.5 \%$ (63 of 120$)$. Five of the 120 patients included did not complete the treatment: in the arginine group, two HIV+ patients died and one $\mathrm{HIV}+$ patient moved to another area; and in the placebo group, one HIV+ patient died and one HIV-patient moved to another area. Protocol was by intention-to-treat but as the drop out rate was low $(4.2 \%$, five of 120$)$ and equally distributed among groups, the authors did not include these patients in the statistical analysis. Of the 115 patients that completed treatment, 57 patients were randomly assigned to the arginine group and 58 to the placebo group, and then further stratified by HIV serology according to protocol (fig. 1). The difference in the proportion of HIV+ patients in the arginine group compared with the placebo group was not 


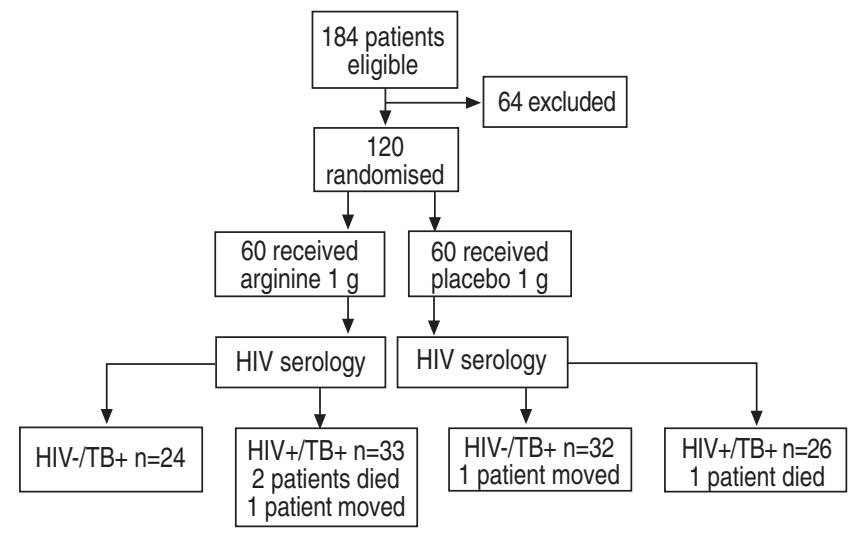

Fig. 1. - Trial profile. HIV: human immunodeficiency virus; TB: tuberculosis.

significant (Chi-squared test). No side-effects were reported in the arginine or placebo groups. There were no significant differences in any of the baseline characteristics between the arginine and the placebo group, including weight, sputum smear result, SR or the number of weeks the patients had been suffering from the various symptoms at baseline (table 1). The seroprevalence of HIV in patients that completed treatment was 51.3\% (59 of 115). At baseline, HIV+/TB+ patients had significantly higher levels of SR values and serum TNF- $\alpha$ than HIV-/TB+ patients, in both the arginine and the placebo group (table 1).

Arginine supplementation induces increased sputum conversion and reduced cough in HIV-negative patients with smear-positive $T B$

Arginine treated HIV-/TB+ patients had significantly increased sputum conversion at week 8 (100\% (24 of 24) versus placebo $84 \%$ (27 of 32)) and a reduced prevalence of cough at week 2 (arginine $67 \%$ (16 of 24) versus placebo $94 \%$
(30 of 32)) and week 8 (arginine $25 \%$ (six of 24) versus placebo $66 \%$ (21 of 32); fig. 2). Double multivariate regression analysis showed a significant difference in primary outcomes (clinical symptoms, weight gain and sputum conversion from baseline to week 8) between the arginine- and placebosupplemented HIV-/TB+ patients (figs 2 and 3 ). The argininesupplemented HIV-/TB+ patients exhibited a more pronounced and rapid reduction in all symptoms and increased sputum conversion at weeks 2 and 8 compared with placebo (fig. 2). There was a tendency for a more rapid increase in weight in the arginine-supplemented HIV-/TB+ patients compared with the placebo group, although this did not reach statistical significance when analysing weight gain as a single variable among the primary outcomes (fig. 3). Considering HIV-l $\mathrm{TB}+$ patients from baseline to week 2 , serum arginine levels increased significantly in the arginine treated $(100.2 \mu \mathrm{M}$ (90.5-109.9) versus $142.1 \mu \mathrm{M}$ (114.1-170.1)) compared with the placebo treated $(105.5 \mu \mathrm{M}(93.7-117.3)$ versus $95.7 \mu \mathrm{M}$ (82.4-108.9); fig. 4). Serum citrulline increased significantly in the HIV-/TB+ subjects receiving arginine $(27.3 \mu \mathrm{M}(23.2-$ 31.5) versus $42.8 \mu \mathrm{M}(35.4-50.2)$ ) compared with placebo (28.2 $\mu \mathrm{M}$ (24.4-31.9) versus $32.8 \mu \mathrm{M}$ (28.6-37.0); fig. 5). The level of urinary NO metabolites in both HIV- and HIV+ patients given arginine decreased from week 0 to week 8 $(2,043 \mu \mathrm{M}(1,607-2,479)$ versus $1,607 \mu \mathrm{M}(1,394-1,820))$ and was significantly lower than in the placebo group at week 8 $(1,964 \mu \mathrm{M}(1,544-2,383)$ for week 0 versus $2,135 \mu \mathrm{M}(1,734$ 2,536) for week 8; fig. 6).

Analysis of the effect of supplementary arginine on a subgroup of patients with low baseline serum arginine levels

HIV-/TB+ patients with baseline serum arginine levels below the total mean $(103.3 \mu \mathrm{M})$ were further analysed as a subgroup. Repeated measures analysis of these patients showed significantly increased sputum conversion and reduction in clinical symptoms in those receiving arginine $(n=13)$ compared with those given placebo $(n=16)$. Four of five HIV-/TB+

Table 1.-Baseline characteristics of tuberculosis (TB) patients treated with supplementary arginine or placebo

\begin{tabular}{|c|c|c|c|c|}
\hline \multirow[t]{2}{*}{ Characteristic } & \multicolumn{2}{|c|}{ HIV-/TB+ } & \multicolumn{2}{|c|}{$\mathrm{HIV}+/ \mathrm{TB}+$} \\
\hline & Arginine & Placebo & Arginine & Placebo \\
\hline Patients $n$ & 24 & 32 & 33 & 26 \\
\hline Age yrs & $28.8(24.4-33.2)$ & $29.9(26.3-33.6)$ & $29.1(26.5-31.7)$ & $30.8(26.7-34.8)$ \\
\hline Sex M/F & $13 / 11$ & $16 / 16$ & $13 / 20$ & $19 / 7$ \\
\hline \multicolumn{5}{|l|}{ Sputum status $\%$} \\
\hline Three positive & 62.5 & 62.5 & 48.5 & 53.9 \\
\hline Two positive & 25 & 25 & 33.3 & 34.6 \\
\hline One positive & 12.5 & 12.5 & 18.2 & 11.5 \\
\hline Weight $\mathrm{kg}$ & $47.8(43.9-51.7)$ & $45.3(42.3-47.2)$ & $45.0(42.5-47.5)$ & $45.3(42.8-48.3)$ \\
\hline $\mathrm{SR} \mathrm{mm} \cdot \mathrm{h}^{-1}$ & $60.4(50.2-70.6)$ & $59.6(51.3-67.9)$ & $89.0(82.0-96.0)^{*}$ & $79.4(67.5-91.3)^{*}$ \\
\hline Haemoptysis weeks & $0.5(0-1.2)$ & $0.5(0.1-0.9)$ & $0.4(0.1-0.7)$ & $0.2(0-0.4)$ \\
\hline Cough weeks & $9.5(7.5-11.4)$ & $9.3(7.6-11.1)$ & $7.6(5.6-9.7)$ & $9.1(6.9-11.4)$ \\
\hline Chest pain weeks & $5.3(2.9-7.7)$ & $5.7(3.8-7.7)$ & $4.5(2.8-6.1)$ & $5.6(3.4-7.8)$ \\
\hline Fever weeks & $4.4(2.7-6.1)$ & $6.7(4.8-8.6)$ & $6.1(4.4-7.8)$ & $5.0(3.6-6.5)$ \\
\hline Night sweating weeks & $5.1(3.2-7.0)$ & $6.3(4.4-8.2)$ & $5.9(4.1-7.8)$ & $4.7(3.1-6.2)$ \\
\hline Arginine $\mu \mathrm{M}$ & $100.2(90.5-109.9)$ & $105.5(93.7-117.3)$ & $112.5(97.4-127.6)$ & $109.0(95.5-123.1)$ \\
\hline Citrulline $\mu \mathrm{M}$ & $27.3(23.2-31.5)$ & $28.2(24.4-31.9)$ & $27.0(23.1-30.9)$ & $24.5(21.4-27.6)$ \\
\hline NOx $\mu \mathrm{M}$ & $1866(1221-2512)$ & 1679 (1295-2062) & $2172(1557-2787)$ & $2314(1491-3139)$ \\
\hline $\mathrm{TNF}-\alpha \mathrm{pg} \cdot \mathrm{mL}^{-1}$ & $5.3(3.5-7.1)$ & $6.8(4.7-9.0)$ & $14.2(9.4-19.1)^{*}$ & $13.5(10.3-16.6)^{*}$ \\
\hline
\end{tabular}

Data are presented as mean (95\% confidence interval). HIV: human immunodeficiency virus; M: male; F: female; SR: sedimentation rate; NOx: nitric oxide metabolites (nitrate and nitrite); TNF: tumour necrosis factor. There were no significant differences in baseline data between the arginine- and placebo-supplemented groups in HIV-/TB+ or HIV+/TB+ patients, respectively. *: p $<0.05$ between HIV- and HIV+ patients. 


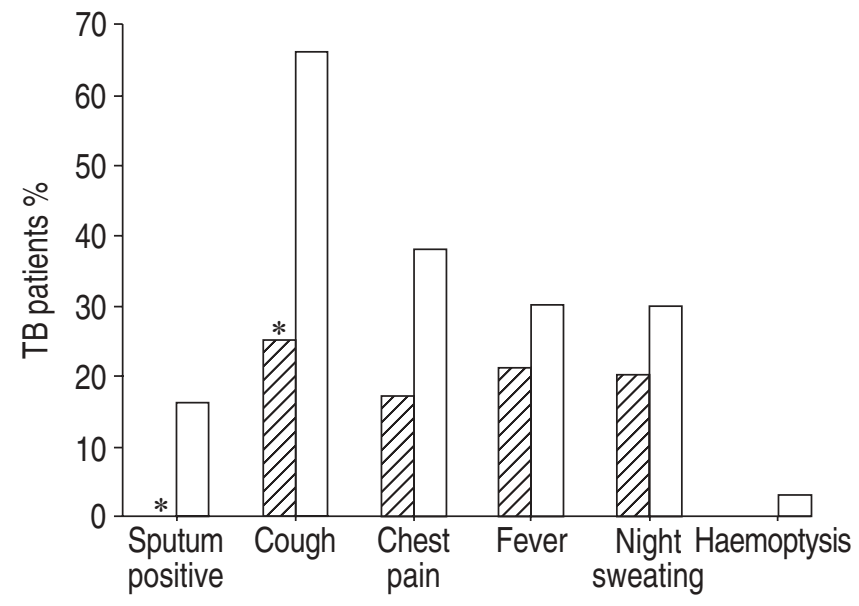

Fig. 2.-Clinical symptoms and sputum conversion at week 8 in human immunodeficiency virus (HIV)- patients with active pulmonary tuberculosis (TB). $\mathbb{Z}$ : HIV- arginine group; $\square$ : HIV- placebo group. $*$ : $\mathrm{p}<0.05$ versus placebo.

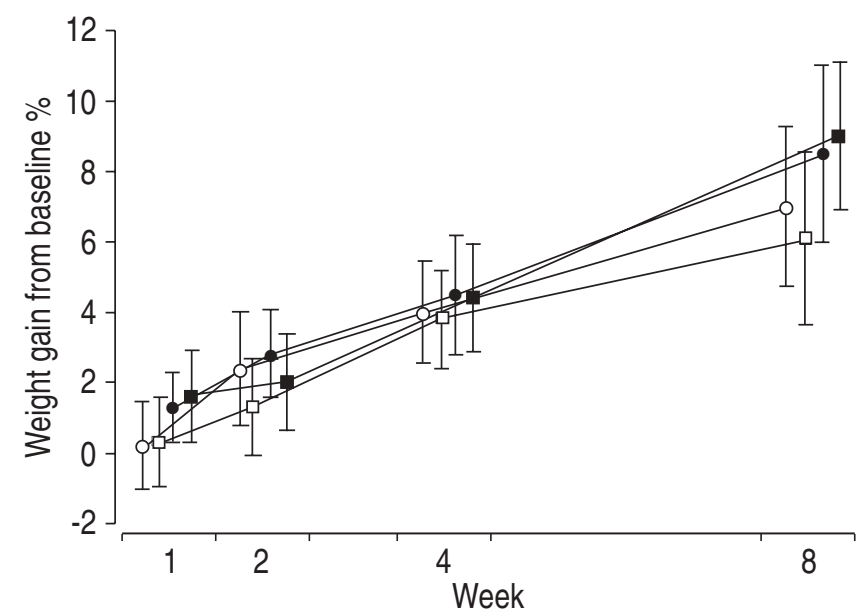

Fig. 3. - Weight gain in human immunodeficiency virus (HIV)-/tuberculosis $(\mathrm{TB})+$ and $\mathrm{HIV}+/ \mathrm{TB}+$ patients treated with supplementary arginine or placebo. Bars represent $95 \%$ confidence intervals. $\bigcirc$ : HIVarginine group; $\mathbf{\square I V}+$ placebo group; $\bullet: \mathrm{HIV}+$ arginine group; $\square$ : HIV- placebo group.

patients that did not sputum convert at week 8 were found in the placebo subgroup with baseline arginine levels below the group mean. At week 2, there was a tendency towards a decrease in NO metabolite levels in the placebo-treated lowserum arginine subgroup $(1,777 \mu \mathrm{M}(1,128-2,424)$ versus $1,474 \mu \mathrm{M}(1,016-1,932))$, whereas levels remained high in the arginine-treated subgroup $(1,747 \mu \mathrm{M}(1,144-2,350)$ versus $1,809 \mu \mathrm{M}(834-2,784))$. HIV-/TB+ patients given arginine showed a significant increase in serum arginine levels from baseline to week $2(84.9 \mu \mathrm{M}(79.4-90.4)$ versus $141.5 \mu \mathrm{M}$ (96.5-186.6)) compared with those given placebo $(82.3 \mu \mathrm{M}$ (71.4-93.2) versus $85.1 \mu \mathrm{M}(67.9-102.2)$ ). In the subgroup of arginine-supplemented $\mathrm{HIV}-/ \mathrm{TB}+$ patients with baseline arginine levels above the mean, no increase in arginine levels, clinical improvement or increased sputum conversion was detected compared with placebo.

Arginine gives no clinical improvement in smear-positive patients co-infected with human immunodeficiency virus

Double multivariate regression analysis of $\mathrm{HIV}+/ \mathrm{TB}+$ patients revealed no significant differences between the

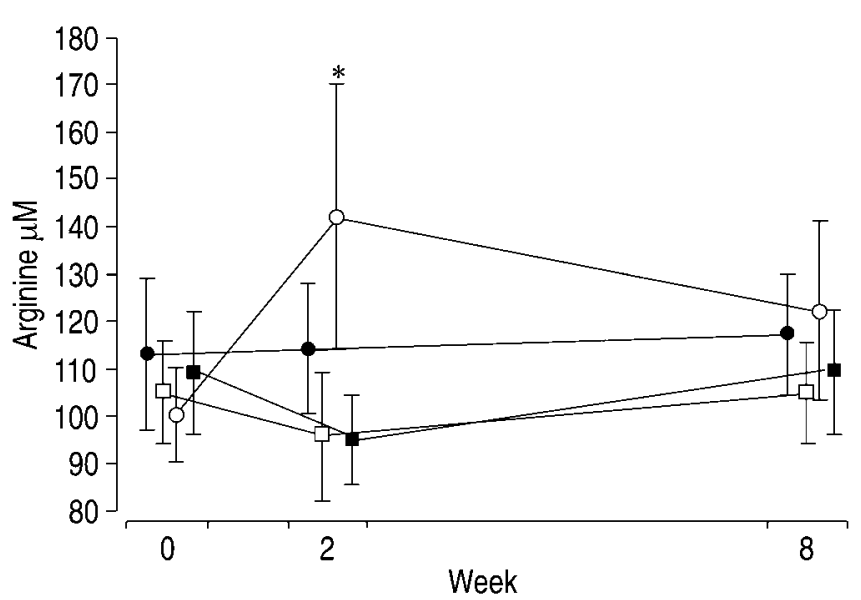

Fig. 4. - Serum arginine levels in human immunodeficiency virus (HIV)-/tuberculosis (TB)+ and $\mathrm{HIV}+/ \mathrm{TB}+$ patients treated with supplementary arginine or placebo. Bars represent 95\% confidence intervals. $\bigcirc$ : HIV- arginine group; $\mathbf{\square}$ : HIV+ placebo group; $\bullet$ HIV+ arginine group; $\square$ : HIV- placebo group. *: $\mathrm{p}<0.05$ between arginine and placebo treatment in HIV-/TB + patients.

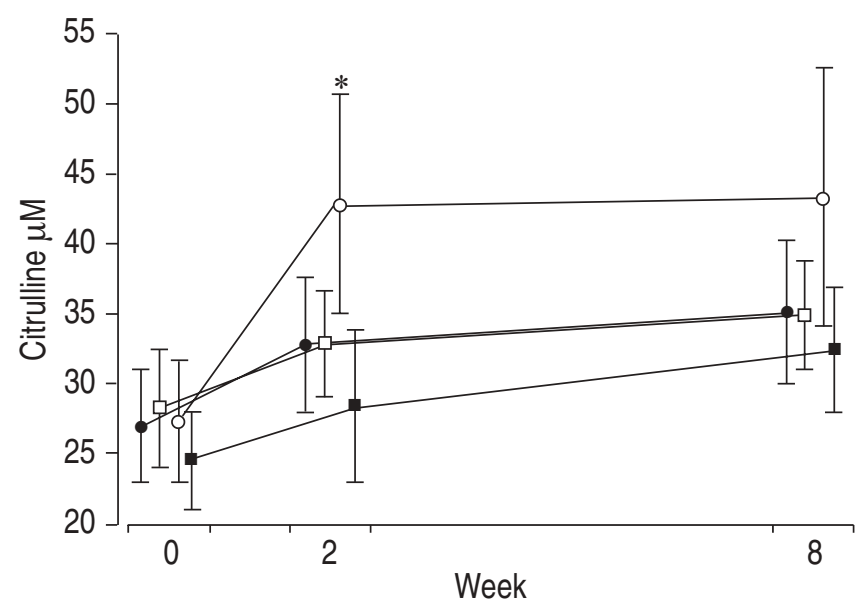

Fig. 5. - Serum citrulline levels in human immunodeficiency virus (HIV)-/tuberculosis (TB) + and $\mathrm{HIV}+/ \mathrm{TB}+$ patients treated with supplementary arginine or placebo. Bars represent $95 \%$ confidence intervals. $\bigcirc$ : HIV-arginine group; $\mathbf{\square}:$ HIV+ placebo group; $\bullet:$ HIV+ arginine group; $\square$ : HIV- placebo group. ${ }^{*}: \mathrm{p}<0.05$ between arginine and placebo treatment in HIV-/TB+ patients.

arginine- and placebo-supplemented groups in regard to primary outcomes (figs 3 and 7) or serum levels of arginine or citrulline (figs 4 and 5). Moreover, arginine treatment had no effect on SR or TNF- $\alpha$ in either HIV+/TB+ patients or HIV-/ $\mathrm{TB}+$ patients (data not shown) and it had no significant impact on primary or secondary outcomes (including serum arginine levels) in $\mathrm{HIV}+/ \mathrm{TB}+$ patients whose baseline serum arginine levels were below or above the mean concentration $(111.1 \mu \mathrm{M})$.

\section{Discussion}

These results show that in HIV-patients with smear-positive $\mathrm{TB}$, arginine supplementation has a significant and favourable effect on weight gain, sputum conversion, and reduction of symptoms like cough. The reduced cough and increased mycobacterial clearance in sputum observed during the first 2 months of DOTS treatment in HIV-/TB+ patients receiving arginine supplementation may have substantial impact on transmission of the disease. The improved clinical outcome was associated with increased arginine levels, which suggests an arginine-mediated antimycobacterial effect. The fact that 


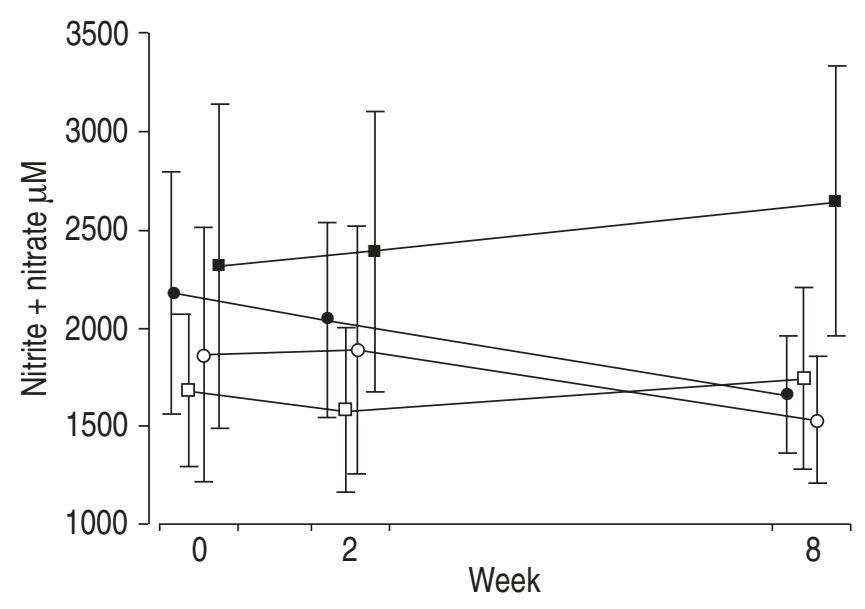

Fig. 6. - Urinary nitric oxide metabolite levels in human immunodeficiency virus (HIV)-/tuberculosis (TB) + and HIV+/TB + patients treated with supplementary arginine or placebo. Bars represent $95 \%$ confidence intervals. $\bigcirc$ : HIV- arginine group; 口: HIV+ placebo group; $\bullet$ : HIV+ arginine group; $\square$ : HIV- placebo group.

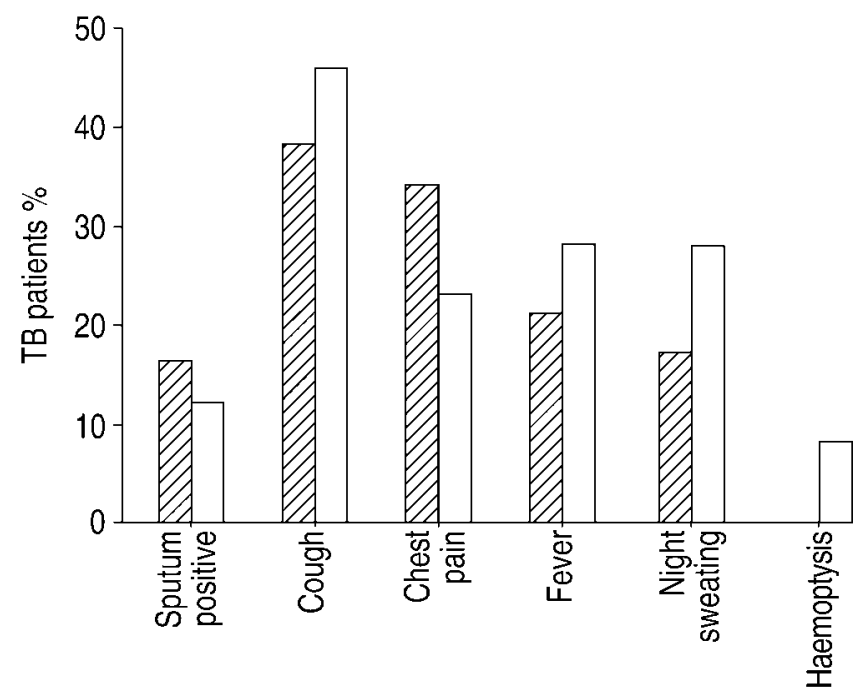

Fig. 7. - Clinical symptoms and sputum conversion at week 8 in human immunodeficiency virus (HIV)+ patients with active pulmonary tuberculosis (TB). $\mathbb{Z}$ : HIV+ arginine group; $\square$ : HIV+ placebo group.

four of five HIV-/TB+ placebo-treated patients that did not sputum convert at week 8 were present in the subgroup with low baseline arginine levels indicates that low arginine levels are associated with an impaired sputum conversion. Moreover, analysis of the subgroup comprising HIV-/TB+ patients with low baseline serum arginine levels showed that after 2 weeks of treatment, concentrations of arginine had markedly increased in those receiving arginine but remained low in those given placebo. It is highly unlikely that the clinical impact of the low dose of arginine used in this study, which is one-fifth of the normal intake [18], is mediated solely by increased protein synthesis. A relatively low dose of arginine $(1 \mathrm{~g})$ for supplementation during a long period avoids the risk of possible side-effects of rapidly replenishing the substrate for iNOS. Previously, $1.5 \mathrm{~g}$ of arginine has been safely administered for 3 months to patients with interstitial cystitis with some positive effects on clinical symptoms [19]. In a previous study conducted in Gondar [20], the arginine level in healthy individuals was $119 \mu \mathrm{M}$, which is similar to normal levels in Europeans [18]. A previous study has shown that arginine supplementation led to decreased morbidity from infectious diseases in high-risk patients undergoing surgery [21].
Nitrite and nitrate, the stable metabolites of NO, can be measured in morning urine as indicators of NO production [22]. It is unlikely that food makes a large contribution to nitrate and nitrite levels in untreated TB patients, since food intake is markedly reduced in patients with this disease. Previous studies have shown that the mean level of NO metabolites is $800-1,100 \mu \mathrm{M}$ in healthy individuals in Ethiopia and Europe [8, 9], but in the present study the authors found baseline levels that were twice as high, indicating that NO production is increased in TB patients. The authors have previously shown, as have others, that iNOS-mediated generation of NO occurs in human macrophages in response to $M$. tuberculosis infection [5-10]. The improved clinical outcome observed in $\mathrm{HIV}-/ \mathrm{TB}+$ patients was probably mediated by augmented production of NO induced by increased arginine intake.

The analysis of the subgroup comprising HIV-/TB+ patients with low baseline serum arginine levels showed that, after 2 weeks of treatment, concentrations of NO metabolites were maintained in those receiving arginine but showed a tendency to decrease in those given placebo. Increased citrulline levels were also observed at week 2 in the arginine group compared with placebo subjects, which suggests increased arginine-mediated generation of NO because citrulline is a metabolite of iNOS-catalysed NO production. Compared with the placebo group, the argininetreated patients exhibited significantly decreased NO levels at week 8 , possibly because arginine supplementation led to locally increased iNOS catalysed production of NO early after treatment initiation, resulting in earlier and greater mycobacterial clearance and thus removal of the stimuli for NO production. These results support a role for arginine supplementation aimed at enhancing the human antimycobacterial defence through increased iNOS-mediated NO production during the early stages of the initial treatment of active TB. However, further studies using more sensitive and frequent measurements of locally produced NO are warranted to explore the mechanism behind arginine-induced clinical improvement of HIV- patients with active TB.

The patients received the chemotherapy recommended by WHO, consisting of isoniazid, rifampicin, pyrazinamide and ethambutol or streptomycin. Use of ethambutol or streptomycin was equally distributed among the groups and did not affect the treatment outcome or the response to arginine. The placebo group had an overall sputum conversion rate of $86 \%$, which suggests good compliance and treatment outcome. Sputum smears were analysed without culture facilities and thus, it cannot be excluded that some patients in this study had atypical mycobacterial infections or were infected with multidrug-resistant strains. However, the presence of atypical mycobacterial infections in smear-positive patients $(1 \%)$ and multidrug-resistance $(1.2 \%)$ in Ethiopia is low, so this probably had little effect on the results [23, 24].

It has been reported that TB accelerates HIV infection because it increases the viral load by inducing persistent immune activation and high levels of TNF- $\alpha[25,26]$. In addition, decreased survival has been observed in HIV+/TB+ compared with HIV-/TB+ patients [27], which is in agreement with the fact that the three patients who died in this study were positive for both HIV and TB. Measurements of serum TNF- $\alpha$ as an indicator of Th1 activation confirmed previously published results, showing significantly higher TNF- $\alpha$ levels in HIV+/TB+ than in HIV-/TB+ patients [26].

The incidence of TB and HIV is high in Ethiopia and seroprevalence in smear-positive TB patients was found to be 49\% in Addis Ababa in $1999[1,28]$ and was 52.5\% in Gondar in this study. Most of the patients studied were probably in the early stages of HIV infection because only sputumpositive individuals were included. This conclusion is supported 
by the high SR values, and TNF- $\alpha$ and NO metabolite levels in these patients, which indicates strong, cell-mediated immunity. A plausible explanation for the observation that arginine supplementation led to clinical improvement in HIV-/TB+ but not HIV+/TB+ patients, is that co-infection with HIV causes more general immune activation, in which arginine is used not only by macrophages in the lungs, but also by such leukocytes at other sites of infection. This hypothesis is supported by the current findings that arginine levels did not significantly increase in the argininesupplemented HIV+ patients, and that these patients also had higher levels of NO metabolites than HIV- patients. HIV is known to induce iNOS-dependent NO production [15], but this might be impaired with decreasing CD4+ cell counts.

Arginine as a supplement to anti-TB chemotherapy given to HIV-/TB+ patients attending the DOTS programme may represent a valuable and cost-effective new treatment strategy that might shorten the duration of conventional chemotherapy by enhancing anti-mycobacterial host defence. Moreover, the enhanced sputum conversion and reduced cough induced by arginine supplementation may reduce the transmission of smear-positive TB. It should be noted that groundnuts (peanuts) contain $1 \mathrm{~g}$ of arginine per $30 \mathrm{~g}$ of biomass and they are affordable and readily available worldwide [29].

To conclude, the authors found that arginine is beneficial as an adjuvant treatment in human immunodeficiency virusnegative patients with active tuberculosis, an effect most likely mediated by increased production of nitric oxide.

Acknowledgements. The authors would like to thank M. Senbeto, T. Mesfin and L. Awoke at the DOTS Clinic GCMS for invaluable help with monitoring patients and collecting patient samples.

\section{References}

1. World Health Organization. Global TB report. Geneva, World Health Organization, 2001

2. Orme IM, Cooper AM. Cytokine/Chemokine cascades in immunity to tuberculosis. Immunol Today 1999; 20: 307-312.

3. Hernández-Pando R, Schön T, Orozco EH, Serafin J, Estrada-García I. Expression of inducible nitric oxide synthase and nitrotyrosine during the evolution of experimental pulmonary tuberculosis. Exp Mol Toxicol 2001; 53: 257-265.

4. Chan J, Tanaka K, Carrol D, Flynn J, Bloom BR. Effects of nitric oxide synthase inhibitors on murine infection with Mycobacterium tuberculosis. Infect Immun 1995; 63: 736-740.

5. Chan ED, Chan J, Schluger NW. What is the role of nitric oxide in murine and human host defense against tuberculosis? Am J Respir Cell Mol Biol 2001; 25: 606-612.

6. Nathan C, Shiloh MU. Reactive oxygen and nitrogen intermediates in the relationship between mammalian hosts and microbial pathogens. Proc Nat Acad Sci USA 2000; 97: 8841-8848.

7. Nicholson S, Bonecini-Almeida M, Lapa e Silva JR, et al. Inducible nitric oxide synthase in pulmonary alveolar macrophages from patients with tuberculosis. J Exp Med 1996; 183: 2293-2302.

8. Choi HS, Rai PR, Chu HW, Cool C, Chan ED. Analysis of nitric oxide synthase and nitrotyrosine expression in human pulmonary tuberculosis. Am J Respir Crit Care Med 2002; 166: $178-186$.

9. Wang CH, Liu CY, Lin HC, Yu CT, Chung KF, Kuo HP. Increased exhaled nitric oxide in active pulmonary tuberculosis due to inducible NO synthase up regulation in alveolar macrophages. Eur Respir J 1998; 11: 809-815.
10. Schön T, Gebre N, Sundqvist T, Aderaye G, Britton S Effects of HIV co-infection and chemotherapy on the urinary levels of nitric oxide metabolites in patients with pulmonary tuberculosis. Scan J Inf Dis 1999; 31: 23-26.

11. Änggård E. Nitric oxide: mediator, murderer and medicine. Lancet 1994; 343: 1199-1206.

12. Reyes AA, Karl IE, Klahr S. Role of arginine in health and renal disease. Am J Physiol 1994; 267: F331-F346.

13. Macallan DC. Malnutrition in tuberculosis. Diagn Microbiol Infect Dis 1999; 34: 153-157.

14. Mogues T, Goodrich ME, Ryan L, LaCourse R, North RJ. The relative importance of $\mathrm{T}$ cell subsets in immunity and immunopathology of airborne Mycobacterium tuberculosis infection in mice. J Exp Med 2001; 193: 271-280.

15. Groeneveld PHP, Kroon FP, Nibbering PH, Bruisten SM, van Sweiten P, van Furth R. Increased production of nitric oxide correlates with viral load and activation of mononuclear phagocytes in HIV-infected patients. Scan J Infect Dis 1996; 28: 341-345.

16. Verdon CP, Burton BA, Priot RL. Sample pretreatment with nitrate reductase and glucose 6 phosphate dehydrogenase quantitatively reduces nitrate while avoiding interference by $\mathrm{NADP}+$ when the griess reation is used to assay for nitrite. Anal Biochem 1995; 224: 502-508.

17. Carlberg M. Assay of neuronal nitric oxide synthase by HPLC determination of citrulline. J Neurosc Met 1994; 52: $165-167$.

18. Cheng JWM, Balwin SN. L-arginine in the management of cardiovascular diseases. Ann Pharmacother 2001; 35: 755-764.

19. Korting GE, Smith SD, Wheeler MA, Weiss RM, Foster HE Jr. A randomized double-blind trial of oral L-arginine for treatment of interstitial cystitis. J Urol 1999; 161: 558565.

20. Peters WH, Lubs H. Serum free amino acids of healthy males and pregnant women from Ethiopia. Die Nahrung 1987; 31: 923-925.

21. Tepaske R, Velthuis H, van Straaten HM, et al. Effect of preoperative oral immune-enhancing nutritional supplement on patients at high risk of infection after cardiac surgery: a randomised placebo-controlled trial. Lancet 2001; 358: 696-701.

22. Kurioka S, Koshimura K, Sugitani M, Murakami Y, Nishiki M, Kato Y. Analysis of urinary nitric oxide metabolites in healthy subjects. Endocr $J$ 1999; 46: 421-428.

23. Abate G. Drug-resistant tuberculosis in Ethiopia: problem scenarios and recommendation. Ethiop Med J 2002; 40: 79-86.

24. Bruchfeld J, Aderaye G, Palme IB, et al. Evaluation of outpatients with suspected pulmonary tuberculosis in a high HIV prevalence setting in Ethiopia: clinical, diagnostic and epidemiological characteristics. Scand J Infect Dis 2002; 34 : 331-337.

25. Toossi Z, Mayanja-Kizza H, Hirsch CS, et al. Impact of tuberculosis on HIV-1 activity in dually infected patients. Clin Exp Immunol 2001; 123: 223-238.

26. Lawn SD, Shattock RJ, Acheampong JW, et al. Sustained plasma TNF- $\alpha$ and HIV-1 load despite resolution of other parameters of immune activation during treatment of tuberculosis in Africans. AIDS 1999; 13: 2231-2237.

27. Harries AD, Hargreaves NJ, Kemp J, et al. Deaths from tuberculosis in sub-Saharan African countries with a high prevalence of HIV-1. Lancet 2001; 357: 1519-1523.

28. Demissie M, Lindtjorn B, Tegbaru B. Human immunodeficiency virus (HIV) infection in tuberculosis patients in Addis Ababa. Eth J Health Dev 2000; 14: 277-282.

29. FAO (Food and Agricultural Organisation, United Nations). Amino acid contents of food and biological data on proteins. FAO Nutrition Studies 24. Rome, Italy, 1970. 